\title{
A SYSTEMATIC SNAPSHOT REVIEW OF CUSTOM-MADE
}

\section{SOFTWARE ENTERPRISES FROM THE}

DEVELOPMENT PERSPECTIVES

\author{
MOUTASM TAMIMI $^{1}$, FATIMAH ALGHAMDI ${ }^{2}$ \& AHID YASEEN ${ }^{3}$ \\ ${ }^{I}$ Department of Software Engineering, College of Information Technology, Zarqa University, Zarqa, Jordan \\ ${ }^{2}$ Department of Information Technology, College of Computing and Information Technology, \\ King Abdulaziz University, Jeddah, Saudi Arabia \\ ${ }^{3}$ Department of Software Engineering, College of Information Technology, Zarqa University, Zarqa, Jordan
}

\begin{abstract}
Nowadays, many organizations are required to develop custom-made software enterprises with its own characteristics by In-house software development companies. It is very complex to deal with custom demands from different environments by a general process from development perspectives. This study attempts to investigate the development perspectives on custom-made software enterprises over the past eleven years. A systematic snapshot methodology was conducted on the gathered published papers between the periods from January 2007 to October 2018. According to the inclusion and exclusion criteria, 102 papers were identified. Reviewed articles addressed 6 primary phases of the software development life cycle (SDLC). Implementation phase was the most commonly used in 35\% of paper's investigations. Case study design was the most common approach used in $26 \%$ of papers. This study presents decisions for trending to future researches based on systematic mapping findings that revealed in gap research forum covering development issues.

KEYWORDS: Literature Review, Custom-Made Software, Development Perspectives \& Systematic Snapshot
\end{abstract}

Received: Dec 22, 2018; Accepted: Jan 12, 2019; Published: Feb 18, 2019; Paper Id.: IJISMRDJUN20191

\section{INTRODUCTION}

Every decade, software engineering researchers produce updated methods in different directions of software development. This guide the In-house software development companies with their team members to deal with different types of software such as packaged software, custom-made software or hybrid software according to follow the produced recourses in development perspectives. It is clear that dealing with packaged software easier than custom-made software in order to the process of eliciting the project requirement engineering. Custom-made software has been produced at In-house at software development companies, which is known as tailor-made software or bespoke software. Custom-made software is considered one of the most critical types of software systems that deals with the challenging diversity of studying, analysing and developing in accordance to customer specific demands, and customization process(Regnell \& Brinkkemper, 2005; Sawyer, 2000). Therefore, In-house software development companies engage in lengthy negotiations with the stakeholders of organizations to develop a specified software requirements(L. Xu \& Brinkkemper, 2007). 
Custom solutions have become popular across organizations, institutes, governments, and other sectors worldwide. These solutions can be either as large enterprises or small enterprises. Based on Laporte et al. investigations of classifying very small enterprises (VSE) the importance of software size determines whether it is used in large projects or SME projects according to the characteristics of their software requirements specifications (SRS) (Laporte, Alexandre, \& O'Connor, 2008), and according to the European Commission (EU) (Commission, 2003) micro, small, and small-to-medium classifications, the importance of the software size can be described whether these enterprises used in large projects or SME projects. For that, not only do large organizations have custom-made solutions but as well small and medium enterprises (SMEs). In SMEs, this software is known as small custom-made software or small bespoke software (Allison, 2010).

The development of custom-made software differs from commercial off-the-shelf (COTS) and packaged software products(Keil \& Carmel, 1995)in terms of that custom-made software meets individual clients and demands, cost of development is expansive more than packaged software, a customized requirement management, untradeable products(Holland, Light, \& Gibson, 1999). It determines the characteristics of management aspects in order to understand tailoring criteria, rationale, and implications of the defined project management criteria(Kalus \& Kuhrmann, 2013). In this context, the purpose of this systematic snapshot review of custom-made software enterprises is to present a wide investigation of literature according to the software development life cycle classifications for discovering the covered research issues and the uncovered issues based on the systematic mapping gaps.

\subsection{Article Structure}

This article is organized as follows; section 2 introduces the recent literature review of the most relevant systematic snapshot studies. Section 3 introduces the material and methods for the systematic mapping process. Section 4 presents the results of the systematic snapshot mapping on the custom-made software with different types of classification schemas. Section 5 shows the findings, discussion. Finally, section 6 concludes the paper content.

\section{LITERATURE REVIEW}

This section summarizes the most relevant contributions of the recently published papers. The reviewed literatures were conducted using the systematic snapshot approach in software engineering (SE) areas.

In 2013, Georg Kalus \& Marco Kuhrmann (Kalus \& Kuhrmann, 2013) conducted a mapping study to specific project requirements called tailor software or custom-made software. It aimed to present a systematic literature survey (SLR) to develop a catalogue that was suitable for the project-specific software in the implication's perspective. This mapping dealt with analysing the impact of the tailored software criteria in the software engineering management by relying on the project characteristics and processes of tailoring software enterprises. In 2018, Tahir et al.(Tahir, Rasool, \& Noman, 2018) conducted a mapping study to investigate the software measurement programs in SMEs for 35 primary studies in order to show the difference between the SMEs and large organizations. It aimed to recognize, explore, and classify the studies on software measurement programs in the period between 2001 and 2016 to the relevant gathered research papers. In 2017, Tamimi, M., \& Jebreen (Tamimi \& Jebreen, 2018) conducted a mapping study of small packaged software with a development perspective interest of 101 research papers between the periods from 2007 to 2017 . Tamimi

\& Jebreen (2018) showed that the contributions of packaged software in SMEs were in these domains: new software development life-cycle for packaged software SMEs, and enterprise resource planning (ERP). Furthermore, Tamimi, M., 
\& Jebreen also (Jebreen, Tamimi, Almajali, \& Janabi, 2018)presented in their 2018 systematic snapshot review the integration testing in enterprise resource planning (ERP) at SMEs to the thirty published papers between the periods from 2013 until 2017. The investigations revealed different classifications. These classifications differed in accordance with the goal investigated by researchers.

However, in this current investigation the researchers aim to introduce an investigation of the custom-made software from an in-house software development perspective.

\section{MATERIAL AND METHOD}

In this context, we conducted and adopted a Systematic Mapping Method (SSM) which is a procedure aims to identify, evaluate and interpret the gathered resources based on a structured strategy that was endorsed by Petersen et al. (Petersen, Feldt, Mujtaba, \& Mattsson, 2008) created this method to develop systematic snapshot approach and to create a classification schema to the fields of software engineering. Moreover, the researchers adhered to the guidelines for the implementation of the systematic literature review and the validation process to ensure rigour of the produced findings.

\subsection{Systematic Mapping Process}

To set the rules of systematic mapping study (SMS) investigation, we carried out a structured systematic mapping process (SMP). This approach was followed to answer specific research questions using a specific research process/approach. Papers' abstracts were searched using specific keywords. This process is described in detail in the next paragraph. Data extraction and mapping is shown in figure 1.

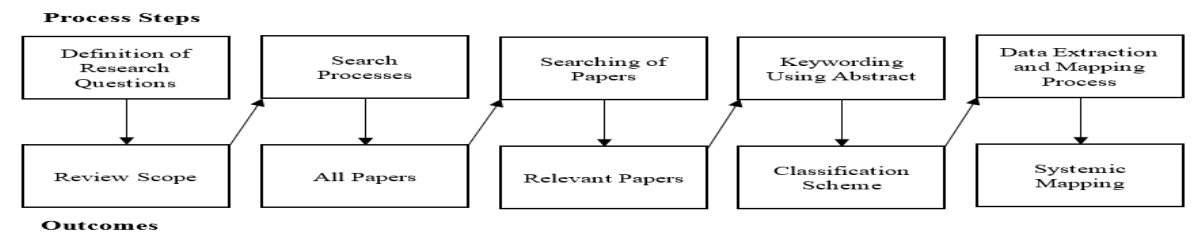

Figure 1: Systematic Mapping Process (Petersen et al., 2008)

\subsubsection{Research Question}

This study concentrates on answering a number of research questions. In fact, investigating and targeting an area in the software engineering does not usually present a new problem domain. The problem domain is identified through understanding the existing resources such as strategies, approaches, models, processes, techniques, and tools. Doing so allows the researchers and industrial practitioners to adapt the state-of-the-art software development systems. For the purpose of presenting the research questions, table 1 shows the primary three Research Questions (RQ) in addition to describe the motivation of each given research question.

Table 1: Research Questions and Motivation

\begin{tabular}{|c|l|l|}
\hline No. & \multicolumn{1}{|c|}{ Research Question } & \multicolumn{1}{|c|}{ Motivation } \\
\hline RQ1 & $\begin{array}{l}\text { What are the investigation fields of custom-made } \\
\text { software In-house software development } \\
\text { companies? }\end{array}$ & $\begin{array}{l}\text { We should first identify the former resources and investigations in different } \\
\text { dimensions in the development aspects. }\end{array}$ \\
\hline RQ2 & $\begin{array}{l}\text { What are the current trends when classifying } \\
\text { custom-made software in the different schemas? }\end{array}$ & $\begin{array}{l}\text { We should second find the software development aspects and analyze } \\
\text { individually each paper and then grouping into the phases of the software } \\
\text { development lifecycle. }\end{array}$ \\
\hline RQ3 & $\begin{array}{l}\text { How is the research being carried out in the } \\
\text { custom-made software in the development } \\
\text { perspectives? }\end{array}$ & $\begin{array}{l}\text { We should finally discuss the findings of classification results to the one } \\
\text { hundred and two relative research papers in order to benefits, research } \\
\text { gap, and limitations. }\end{array}$ \\
\hline
\end{tabular}




\subsubsection{Search Process}

In this part, we addressed the literature search process to enable the researchers to produce a review protocol that ensures the validity and reliability of resources. There is a clear mechanism to conducting the review protocol according to the Petersen approach (Petersen et al., 2008). We followed a structured strategy of the combined. Collected keywords that are intended to inform the scope of this study. Initially, the main keywords used in the literature review of this study concentrated on using the custom software which is also known as bespoke software or tailor software. The researchers used the keywords custom software OR bespoke software OR tailor software to search the database. To identify whether the research was conducted in large development organizations or small development organizations, we used the keywords (Large OR Small OR SMEs). For the development aspects of the In-house software development companies, we used the keywords (development OR strategies OR approaches OR models OR processes OR technique OR tool OR factors). The combination of these keyword groups was further combined using the "AND" and "OR" operators to create the search string and employ them in the search engines of database libraries as shown in figure 2.

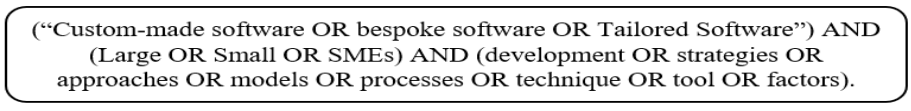

Figure 2: List of Keywords Used in the Search Strings

We carried out the search strings on several data sources were carefully scanned to retrieve the useful studies that satisfy the scope of our study. Primarily, we used web search engines that have scientific digital libraries such as ACM, Springer Link, IEEE Xplore, Science Direct Elsevier, IJEIS, and Wiley to gather relevant articles.

\subsubsection{Screening of Relevant Papers}

This step was utilized to screen the relevant papers that were obtained by a particular domain in the established inclusion and exclusion criteria. These processes were intended to confirm whether the shortlisted papers should be accepted or rejected by checking the inclusion research criteria with the characteristics of the gathered articles. For that purpose, the inclusion criteria identifies as follow; (a) the focus of the studies' abstract and paper is compatible with keywords of these strings (development, model, framework, tool, challenges, problem, improvements, techniques, approaches, lessons, and practices), (b) the studies should address the large or SMEs context, (c) the studies' research methodologies include (survey, case study, experiment, and others), (d) the studies are produced in English language. On the other hand, the exclusion criteria were as follows; (a) the studies are not presented in full-text, (b) the studies are not produced in the English language, (c) the studies are not relevant to the economic and financial perspectives, (d) the studies are not books, presentations, or posters.

\subsubsection{Data Extraction and Mapping Process}

In light of the previous systematic mapping processes, we started extracting the research papers to be accepted or rejected for the scope of our investigations. We collected the 1,023 papers in the initial search and applied the inclusion/exclusion criteria, which resulted in the 179 extracted papers. Then, we rejected 66 papers after conducting the whole text review. 13 more papers were rejected after conducting seek full text. The total of 102 papers were accepted in the mapping processes as shown in the figure 3 


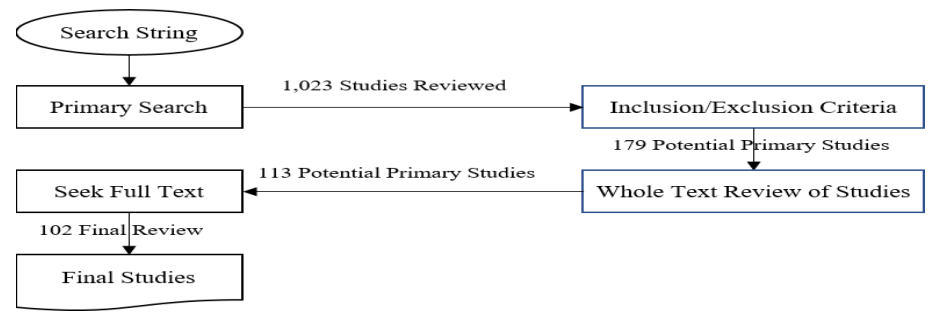

Figure 3: Data Extraction and Mapping Process Results

\section{RESULTS}

This section presents the findings of the systematic snapshot review conducted on one hundred and two custommade software papers produced between the periods from 2007 to 2018. Results were obtained after dealing with the repository of relevant gathered papers, building suitable classification schemas and analysing each relevant paper in the repository according to the different types of the classification schemas. Primarily, the main classification schema is arranged according to the software development life-cycle (SDLC) phases, factors, and issues. In addition, issues such as software size categories (large or small), target countries, research method type, publishers, article types, year of publication and custom-made application names classification schemas were taken into consideration.

\subsection{Aggregating the Classification Schemas}

Building a schema is defined as establishing a protocol strategy to construct a different kind of classification. The followed processes of aggregating the classification schemas in this research were that adopted from Petersen, Kai, et al. (Petersen et al., 2008) as shown in figure 4. In this context, we concentrated on the keywords that were relevant to the development fields in addition to validating the gathered papers within the scope of this research interest. After validating the set of keywords, we analysed the papers that search process yielded to help the researcher to establish previous research contribution and understand the research objectives by aggregating different kind of categories.

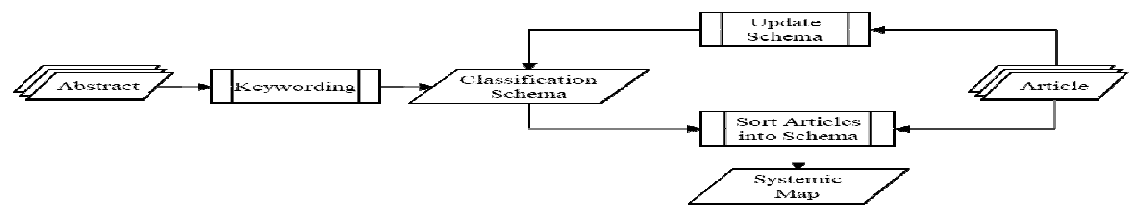

Figure 4: Classification Scheme Aggregation Processes (Petersen et al., 2008).

The gathered papers were analysed in a set of clustered categories. we aggregated the new categories to build new classification schemas that were compatible with SDLC (Planning, design, development, testing, deployment, and maintenance) phases, factors, software size, software applications, publication year, publishers and articles indexed by, as shown in table 2 .

Table 2: Final Set of 9 Associated Classification Schemas

\begin{tabular}{|l|l|l|}
\hline \multicolumn{1}{|c|}{ SDLC } & \multicolumn{1}{|c|}{ Software Size } & \multicolumn{1}{|c|}{ Software Applications } \\
\hline Phases; & & Management applications, \\
Planning, & & E-Commerce applications, \\
Design, & Large, & Mobile applications, \\
Implementation, & Small-and-medium enterprises (SMEs) & Web applications, \\
Testing, & & Widows form applications, \\
Deployment, & & Cloud applications, \\
Maintenance. & & Tool applications, \\
\hline
\end{tabular}




\begin{tabular}{|l|l|l|}
\hline $\begin{array}{l}\text { Factors; } \\
\text { Sub-factors; }\end{array}$ & & And others. \\
\hline Year of publish & Publishers & Articles indexed \\
\hline Targeted countries & Top articles citations & Research methodologies \\
\hline
\end{tabular}

\subsection{Software Development Life Cycle Findings}

According to the importance of software development based on SDLC, this paper presented a vast analysis of the collected 102 papers that were focused on custom-made software development perspectives. The parts of analyses included the planning phase, design phase, development phase, testing phase, deployment phase, and maintenance phase. It also included 15 important factors in management, organization, strategic, and technological issues as shown in the next parts of this section.

\subsubsection{Planning Phase Classification Findings}

The initial phase of the SDLC intended to develop a project charter, project plan, resource plan, financial plan, quality plan, risk plan, acceptance plan, communications plan and a procurement plan. In fact, this phase is very critical to study the requirements negotiated with the clients in the domain of business functional and non-functional requirements. This phase forms the roles and responsibilities between the stakeholders, authorities of the identified stakeholders. Table 3 presents the results of one hundred and two relevant analysed articles.

Table 3: Classifications Based on Planning Phase

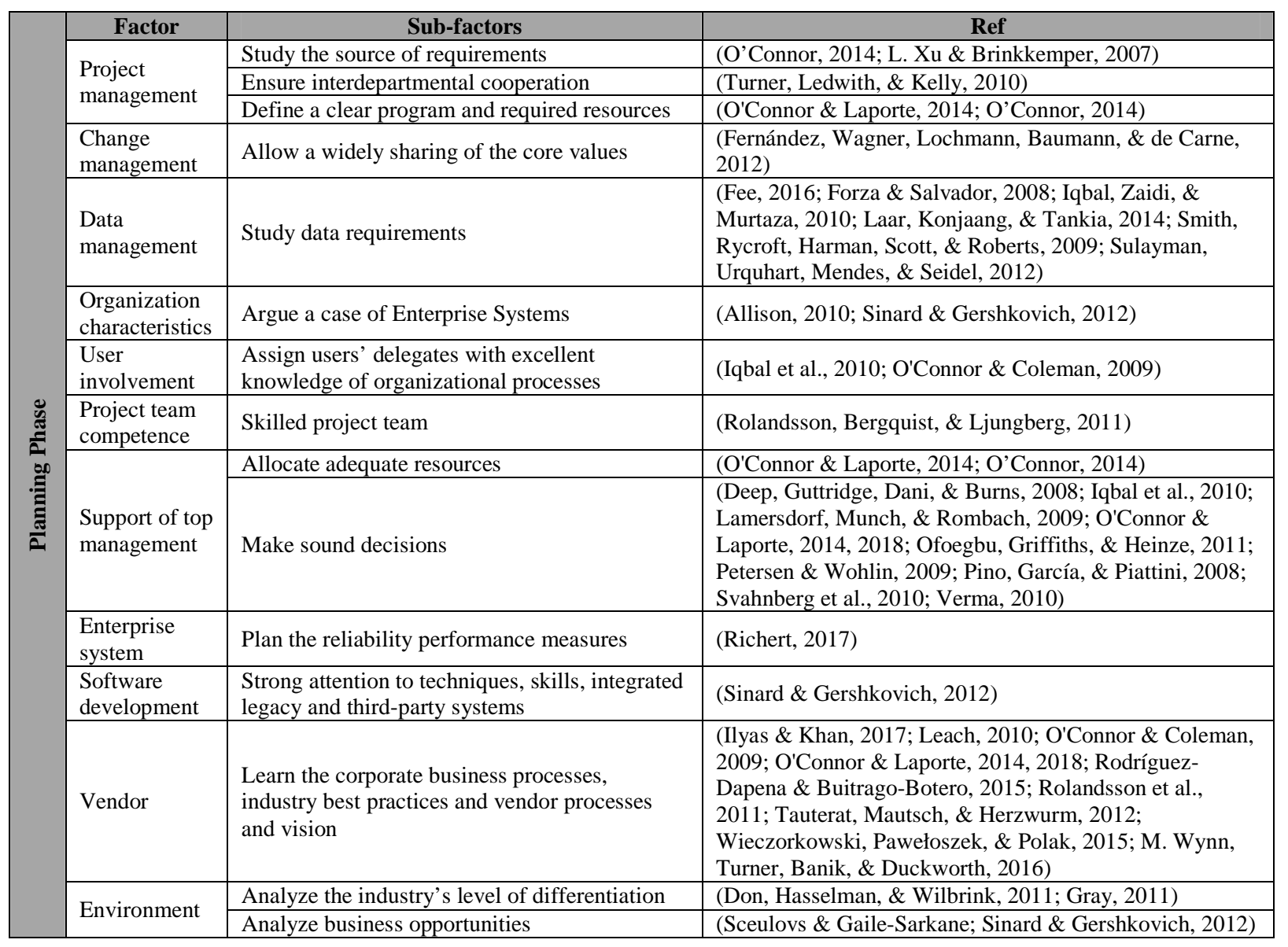




\subsubsection{Design Phase Classification Findings}

System design is a crucial phase to address the identified design requirements that informs the material processes of software engineering architectures, models, frameworks and other system design aspects. This phase clearly affects the structure and software behaviours such as organization of the developed software system. These system behaviours, including Model-driven engineering (MDD, MDE, UML), flowcharts, Data Flow Diagram (DFD), Entity Relational (ER), etc., shape an organized process to accomplish the system design phase. Table 4 presents the analysis of the one hundred and two relevant articles to reveal the discovered 19 research papers in this phase.

Table 4: Classifications Based on Design Phase

\begin{tabular}{|l|l|l|l|}
\hline \multicolumn{1}{|c|}{ Factor } & \multicolumn{1}{|c|}{ Issues } & \multicolumn{1}{c|}{ Ref } \\
\hline \multirow{2}{*}{ Project management } & Follow standard architectures & $\begin{array}{l}\text { (Dzamashvili Fogelström, Gorschek, Svahnberg, \& } \\
\text { Olsson, 2010; Kath, Schreiner, \& Favaro, 2009) }\end{array}$ \\
\cline { 2 - 4 } & Follow model based implementation & (Dzamashvili Fogelström et al., 2010; Kath et al., 2009) \\
\hline \multirow{2}{*}{ Change management } & $\begin{array}{l}\text { Change unclear software architectural } \\
\text { representations }\end{array}$ & (Scacchi \& Alspaugh, 2017) \\
\hline \multirow{2}{*}{ Data management } & Study up-front architecture planning & (Waterman, Noble, \& Allan, 2013) \\
\cline { 2 - 4 } & Study previous systems design data & (Laar et al., 2014) \\
\cline { 2 - 4 } $\begin{array}{l}\text { Strategy and } \\
\text { methodology }\end{array}$ & Contrast with design pattern & (Fernández et al., 2012) \\
\hline \multirow{2}{*}{$\begin{array}{l}\text { Education and } \\
\text { training }\end{array}$} & Improve design guidance, practices & $\begin{array}{l}\text { (Bass, 2016; Gorschek, Tempero, \& Angelis, 2014; } \\
\text { Kruchten, 2013; Lang \& Fitzgerald, 2007) }\end{array}$ \\
\cline { 2 - 4 } & $\begin{array}{l}\text { Study contextual aspects for } \\
\text { architectures software development }\end{array}$ & (Kruchten, 2013) \\
\hline \multirow{2}{*}{$\begin{array}{l}\text { Enterprise system } \\
\text { selection process }\end{array}$} & $\begin{array}{l}\text { Ensure usage a high-level of standards } \\
\text { (Artz, Van De Weerd, \& Brinkkemper, 2010; Bass, } \\
\text { 2016; Deep et al., 2008; O'Connor \& Laporte, 2014, } \\
\text { 2018; O'Connor, 2014) }\end{array}$ \\
\hline \multirow{2}{*}{$\begin{array}{l}\text { Software } \\
\text { development }\end{array}$} & $\begin{array}{l}\text { Studying current product re-design } \\
\text { impact }\end{array}$ & $\begin{array}{l}\text { Strong attention to communities through } \\
\text { a system architecture and template } \\
\text { design }\end{array}$ & $\begin{array}{l}\text { (Perumal, Sulaiman, Sharif, Ramli, \& Leong, 2013; } \\
\text { Rauschecker et al., 2011; Smith et al., 2009) }\end{array}$ \\
\hline
\end{tabular}

\subsubsection{Implementation Phase Classification Findings}

The implementation process is used to reflect the requirements and design phase specification processes that are coded using specific programming language. This phase produces the running systems that meet customer specific requirements that were accurately specified in the contracts. This phase emphasizes the importance of following the managerial, organizational, and technical factors that inform the part development in the practice excellence. Table 5 resulted in analysing the gathered one hundred and two relative papers to reveal the discovered 37 research papers in this phase. 
Table 5: Classifications Based on Implementation Phase

\begin{tabular}{|c|c|c|c|}
\hline & \multirow{2}{*}{$\begin{array}{c}\text { Factor } \\
\text { Project management }\end{array}$} & \multirow{2}{*}{$\begin{array}{ll} & \text { Issues } \\
\text { Manage conflicts }\end{array}$} & \multirow{2}{*}{$\begin{array}{c}\text { Ref } \\
\text { (L. Xu \& Brinkkemper, 2007) }\end{array}$} \\
\hline & & & \\
\hline \multirow{14}{*}{ 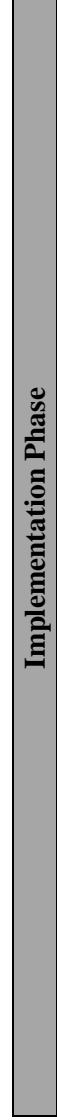 } & \multirow{2}{*}{ Change management } & Develop business justification & $\begin{array}{l}\text { (Abeywardena \& De Coster; Duhan, 2007; } \\
\text { Sceulovs \& Gaile-Sarkane) }\end{array}$ \\
\hline & & $\begin{array}{l}\text { Have a “buy-in” by all major stakeholder's } \\
\text { strategy }\end{array}$ & (Sinard \& Gershkovich, 2012) \\
\hline & Data management & Plan data model & (Forza \& Salvador, 2008) \\
\hline & \multirow{3}{*}{$\begin{array}{l}\text { Strategy and } \\
\text { methodology }\end{array}$} & Develop uncertainties handling methodology & (Duhan, 2007) \\
\hline & & Determine hardware standardization & (Farooque et al., 2013) \\
\hline & & $\begin{array}{l}\text { Make middleware approach, switching } \\
\text { module }\end{array}$ & (Perumal et al., 2013) \\
\hline & \multirow[b]{2}{*}{ Monitoring } & Monitor project progress & (P. Xu \& Ramesh, 2008) \\
\hline & & Develop performance measures & $\begin{array}{l}\text { (Ivarsson, 2018; Ramasubbu, Cataldo, Balan, \& } \\
\text { Herbsleb, 2011) }\end{array}$ \\
\hline & Education and training & Develop users training programs & (Laakso, 2017; Laporte et al., 2008) \\
\hline & Enterprise system & Consist on best practices & $\begin{array}{l}\text { (Alamdy \& Osman, 2017; Gorschek, Gomes, } \\
\text { Pettersson, \& Torkar, 2012; Laporte et al., 2008; } \\
\text { Leach, 2010; O'Connor \& Coleman, 2009; Pino } \\
\text { et al., 2008; Rodríguez-Dapena \& Buitrago- } \\
\text { Botero, 2015; Smith et al., 2009; Tomiwa, 2015; } \\
\text { Wagner, 2017) }\end{array}$ \\
\hline & $\begin{array}{l}\text { Enterprise system } \\
\text { selection process }\end{array}$ & $\begin{array}{l}\text { Make continues justification of additional } \\
\text { functionality through applications }\end{array}$ & $\begin{array}{l}\text { (Fee, 2016; Khurum \& Gorschek, 2011; Matar, } \\
\text { 2015) }\end{array}$ \\
\hline & \multirow[b]{2}{*}{ Software development } & Technical response mechanism & $\begin{array}{l}\text { (Ciolkowski, Heidrich, Simon, \& Radicke, } \\
\text { 2008; Farooque et al., 2013; Fee, 2016; } \\
\text { Papaloizou \& Komodromos, 2009; Perumal et } \\
\text { al., 2013) }\end{array}$ \\
\hline & & Appropriate modelling methods & $\begin{array}{l}\text { (Akbar, Hassan, \& Abdullah, 2012; Bonomi } \\
\text { Santos \& Spring, 2013; Dick, Kern, } \\
\text { Drangmeister, Naumann, \& Johann, 2011; } \\
\text { Lingannavar \& Yammiyavar; Paulson, Sobester, } \\
\text { \& Scanlan, 2017; Richert, 2017; Schubert \& } \\
\text { Merian-Str, 2015) }\end{array}$ \\
\hline & Vendor & Employ IT vendors' skilled representatives & (Aduamoah, 2017; Ilyas \& Khan, 2015) \\
\hline
\end{tabular}

\subsubsection{Testing Phase Classification Findings}

The testing phase is highly necessary to be utilized during and after building a software system in order to achieve a diverse organized level of testing types such as unit testing, integration testing, system testing, and acceptance testing. These types of testing ensure the correctness of the system functionality before delivering the software to the customers that must be satisfied with given functionalities. Table 6 resulted in analysing the gathered one hundred and two relative papers to reveal the discovered 23 research papers in this phase.

Table 6: Classifications based on Testing Phase

\begin{tabular}{|c|c|c|c|}
\hline \multirow{8}{*}{ 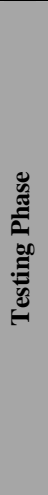 } & Factor & Issues & Ref \\
\hline & \multirow{3}{*}{ Project management } & $\begin{array}{l}\text { Emphasis on a test plan to end of a } \\
\text { project. }\end{array}$ & (Bass, 2016; Ilyas \& Khan, 2017) \\
\hline & & Knowledge transfer & $\begin{array}{l}\text { (Artz, Van De Weerd, \& Brinkkemper, 2010; Gorschek et al., 2012; } \\
\text { Iqbal et al., 2010; M. Wynn \& Turner, 2013; M. G. Wynn, Shen, \& } \\
\text { Brandao, 2008) }\end{array}$ \\
\hline & & $\begin{array}{l}\text { Engaging the 'pragmatic satisficing' } \\
\text { with 'tried-and-tested' solution }\end{array}$ & (Lang \& Fitzgerald, 2007) \\
\hline & Change management & $\begin{array}{l}\text { Enhance "performance priority" policy } \\
\text { rather than "seniority priority" }\end{array}$ & $\begin{array}{l}\text { (Aslan, Stevenson, \& Hendry, 2015; Ciolkowski et al., 2008; } \\
\text { Ramasubbu et al., 2011) }\end{array}$ \\
\hline & User involvement & Break old habits of manual work & $\begin{array}{l}\text { (Arpaia, De Matteis, \& Inglese, 2015; Parry, Rowley, Jones, \& Kupiec- } \\
\text { Teahan, 2012) }\end{array}$ \\
\hline & \multirow{2}{*}{ Environment } & $\begin{array}{l}\text { suitable decision criteria for analyzing } \\
\text { testing software systems }\end{array}$ & (Arpaia et al., 2015) \\
\hline & & $\begin{array}{l}\text { Analyze problems resulting from lack of } \\
\text { integration }\end{array}$ & (Barton \& Thomas, 2009) \\
\hline
\end{tabular}


Enterprises from the Development Perspectives

\begin{tabular}{|l|l|l|l|}
\hline Enterprise system & $\begin{array}{l}\text { Ongoing stream of upgrades to fix bugs, } \\
\text { regulations and new functionality }\end{array}$ & (Sinard \& Gershkovich, 2012) \\
\hline \multirow{2}{*}{ Vendor } & $\begin{array}{l}\text { Analyze vendor's ongoing and future } \\
\text { changes }\end{array}$ & (Saqib, Jan, Ahmad, Ahmad, \& Asghar, 2011) \\
\hline development & $\begin{array}{l}\text { Arrange the internal testing, in house } \\
\text { testing, and external testing }\end{array}$ & (L. Xu \& Brinkkemper, 2007) \\
\cline { 2 - 4 } & $\begin{array}{l}\text { Practicing testing efforts on the technical } \\
\text { challenges }\end{array}$ & (Fernández et al., 2012; P. Xu \& Ramesh, 2008) \\
\cline { 2 - 3 } & Testing a measurement method & (Arpaia et al., 2015; Dick et al., 2011) \\
\hline Monitoring & $\begin{array}{l}\text { Analyze feedback from clients through } \\
\text { beta tests }\end{array}$ & (Aranda, Easterbrook, \& Wilson, 2007; L. Xu \& Brinkkemper, 2007) \\
\hline \multirow{2}{*}{$\begin{array}{l}\text { Enterprise system } \\
\text { selection process }\end{array}$} & $\begin{array}{l}\text { Emphasize to support technologies of } \\
\text { testing tools. }\end{array}$ & $\begin{array}{l}\text { Component/Unit Testing prior to } \\
\text { integration Testing }\end{array}$ & (Ilyas \& Khan, 2015, 2017) \\
\hline
\end{tabular}

\subsubsection{Deployment Phase Classification Findings}

This phase is intended to inform the software system release as a finished product to the customers after practicing different types of software testing. In brief, this phase presents a software system after successfully completing each type of testing to be ready to go live. Therefore, this phase focuses on the principles, strategies, models of system release, best practices to release the systems and lessons of former implementation of the release. Table 7 resulted in analysing the gathered one hundred and two relative papers to reveal the discovered 32 research papers in this phase.

Table 7: Classifications Based on Deployment Phase

\begin{tabular}{|c|c|c|c|}
\hline & Factor & Issues & Ref \\
\hline & & Choosing a proper delivery approach & (L. Xu \& Brinkkemper, 2007) \\
\hline & $\begin{array}{l}\text { Project } \\
\text { management }\end{array}$ & $\begin{array}{l}\text { Analyze objectives and expected outcomes in } \\
\text { light of existing ES, corporate strategy, legacy } \\
\text { system, future standardization and regulations }\end{array}$ & $\begin{array}{l}\text { (Allison, 2010; Haug, Ladeby, \& Edwards, 2009; Jones \& Rowley, } \\
\text { 2009; Kalus \& Kuhrmann, 2013; Mishra \& Mishra, 2009; } \\
\text { Savolainen, Ahonen, \& Richardson, 2012; Sceulovs \& Gaile- } \\
\text { Sarkane; Wieczorkowski et al., 2015) }\end{array}$ \\
\hline & $\begin{array}{l}\text { Change } \\
\text { management }\end{array}$ & Study former obstacles & (Haug et al., 2009; Mateer \& Jones, 2018) \\
\hline & Data management & $\begin{array}{l}\text { Define applications needed for data } \\
\text { management }\end{array}$ & (Arpaia et al., 2015; Smith et al., 2009) \\
\hline & $\begin{array}{l}\text { Organization } \\
\text { characteristics }\end{array}$ & $\begin{array}{l}\text { Ensure the coordination and cooperation of all } \\
\text { stakeholders affected by new module } \\
\text { implementation }\end{array}$ & (Fabriek, van den Brand, Brinkkemper, Harmsen, \& Helms, 2008) \\
\hline & & Develop high level of usage and efficacy & $\begin{array}{l}\text { (Johansson, Deliallisi, \& Walraven, 2016; Khurum \& Gorschek, } \\
\text { 2011) }\end{array}$ \\
\hline 造 & $\begin{array}{l}\text { Project team } \\
\text { competence }\end{array}$ & $\begin{array}{l}\text { Strengthen the project team with relevant to } \\
\text { module personnel and consultants }\end{array}$ & (Pino et al., 2008) \\
\hline ᄅ를 & Strategy and & $\begin{array}{l}\text { Analyze strategy in light of previous } \\
\text { implementations }\end{array}$ & $\begin{array}{l}\text { (Fabriek et al., 2008; Ramasubbu et al., 2011; Rolandsson, } \\
\text { Bergquist, \& Ljungberg, 2009; Rolandsson et al., 2011; Sulayman } \\
\text { et al., 2012; P. Xu \& Ramesh, 2008) }\end{array}$ \\
\hline อั๊ & & $\begin{array}{l}\text { Increase best practices used in security } \\
\text { defenses }\end{array}$ & (Pan \& Fung, 2011) \\
\hline & Monitoring & $\begin{array}{l}\text { Define monitoring measures for unsolved } \\
\text { obstacles that affect new module } \\
\text { implementation }\end{array}$ & (Arpaia et al., 2015) \\
\hline & Fducation and & Enhance users' knowledge and efficacy & (Fricker, 2012) \\
\hline & training & $\begin{array}{l}\text { Train on future interfaces, obsolete legacy } \\
\text { systems, data quality, etc. }\end{array}$ & (Scacchi \& Alspaugh, 2017) \\
\hline & Enterprise system & Study lessons of former implementation & $\begin{array}{l}\text { (Bailetti, 2012; Ciolkowski et al., 2008; Fafoutis, Elsts, Piechocki, } \\
\text { \& Craddock, 2018; Santos et al., 2007; Sundaresan, Burnett, } \\
\text { Feamster, \& De Donato, 2014) }\end{array}$ \\
\hline & & $\begin{array}{l}\text { Performing post implementation gap analysis } \\
\text { of the current fit application }\end{array}$ & (Aslan et al., 2015; Matar, 2015) \\
\hline & $\begin{array}{l}\text { Enterprise system } \\
\text { selection process }\end{array}$ & $\begin{array}{l}\text { Analyze different modules implementation } \\
\text { considering multiple vendors and } \\
\text { infrastructure considerations }\end{array}$ & (Jing \& Yang, 2015) \\
\hline
\end{tabular}




\begin{tabular}{|l|l|l|}
\hline \multirow{2}{*}{ Environment } & $\begin{array}{l}\text { Analyze trading partner readiness to integrate } \\
\text { its business }\end{array}$ & (Barton \& Thomas, 2009) \\
\cline { 2 - 3 } & $\begin{array}{l}\text { Analyze own ability to alter new rules of } \\
\text { competition and leverage new ways to } \\
\text { outperform rivals }\end{array}$ & (Bailetti, 2012) \\
\hline
\end{tabular}

\subsubsection{Maintenance Phase Classification Findings}

This phase is intended to deal with the issues of system repairs and upgrade. It is concerned with maintaining the legacy or existing systems that have higher possible risks to retiring these systems. It is also concerned with issues regarding system upgrades in accordance with the vendor support to meet the updates in the management, organization, development, and cultural changes. This phase supports different strategies, models, frameworks and other development perspectives to understand the ability to upgrade and maintain a software system. Table 8 resulted in analysing the gathered one hundred and two relative papers to reveal the discovered 23 research papers in this phase.

Table 8: Classifications Based on Maintenance Phase

\begin{tabular}{|c|c|c|c|}
\hline & Factor & Issues & Ref \\
\hline & Project management & $\begin{array}{l}\text { Align expectations of capability, complexity, } \\
\text { knowledge gap, barriers removal }\end{array}$ & $\begin{array}{l}\text { (Barton \& Thomas, 2009; Kuivalainen, Lindqvist, } \\
\text { Saarenketo, \& Äijö, 2007; Waterman et al., 2013) }\end{array}$ \\
\hline & Change management & $\begin{array}{l}\text { Organizational understanding of the importance of } \\
\text { infrastructure upgrades }\end{array}$ & (Mijnhardt, Baars, \& Spruit, 2016) \\
\hline & & $\begin{array}{l}\text { Analyze the experience gained throughout initial } \\
\text { implementation }\end{array}$ & (Lamersdorf et al., 2009; Saqib et al., 2011) \\
\hline & $\begin{array}{l}\text { Urganization } \\
\text { characteristics }\end{array}$ & Analyze specific organizational challenges & $\begin{array}{l}\text { (Bonomi Santos \& Spring, 2013; Ilyas \& Khan, } \\
\text { 2017; Lamersdorf et al., 2009; Mijnhardt et al., } \\
\text { 2016; Sundaresan et al., 2014; Verma, 2010) }\end{array}$ \\
\hline$\underset{\Xi}{\pi}$ & Strategy and & Make use of experts & $\begin{array}{l}\text { (Artz, van de Weerd, Brinkkemper, \& Fieggen, } \\
\text { 2010; Deshpande \& Richardson, 2009) }\end{array}$ \\
\hline छ & methodology & Understand potential upgrade issues & $\begin{array}{l}\text { (Hrubeš; Johansson et al., 2016; Salem, 2012; } \\
\text { Sundaresan et al., 2014) }\end{array}$ \\
\hline 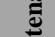 & Monitoring & Ensure former users' satisfaction & (Dzamashvili Fogelström et al., 2010) \\
\hline. & VIonitoring & Ensure end to end operation & (Dzamashvili Fogelström et al., 2010) \\
\hline$\sum^{\pi}$ & Enterprise system & Analyze implications on current processes & (P. Xu \& Ramesh, 2008) \\
\hline & Enterprise system & $\begin{array}{l}\text { Carefully select infrastructure upgraded elements in } \\
\text { light of technical or business improvements needs }\end{array}$ & $\begin{array}{l}\text { (Don et al., 2011; Mishra \& Mishra, 2009; } \\
\text { Rauschecker et al., 2011; Salem, 2012; Sundaresan } \\
\text { et al., 2014; Tamimi, 2018) }\end{array}$ \\
\hline & & Analyze ease of maintenance efforts & $\begin{array}{l}\text { (Don et al., 2011; Hrubeš; Saqib et al., 2011; } \\
\text { Verma, 2010) }\end{array}$ \\
\hline & Software development & $\begin{array}{l}\text { Analyze the benefits of reducing the existing } \\
\text { customizations, enhancements and operational cost }\end{array}$ & (Ballsun-Stanton, Ross, Sobotkova, \& Crook, 2018) \\
\hline & Vendor & Usage of infrastructure modelling tools & $\begin{array}{l}\text { (Costache, Kalus, \& Kuhrmann, 2011; Smith et al., } \\
\text { 2009) }\end{array}$ \\
\hline & Environment & Analyze changes in environmental uncertainties & (Sundaresan et al., 2014) \\
\hline
\end{tabular}

\subsubsection{Systematic Snapshot Mapping Findings}

After classifying the collected 102 papers based on SDLC phases, factors, and sub-factors, we demonstrated the perspectives of development for the custom-made software using a bubble chart to display the number of used research papers for each SDLC phases and factors. Figure 5 summarized our primary results by illustrating the founded contributions, trends, and gaps among the analysed studies. It shows that the number of research papers have heavily focused on support of top management, vendor, data management, and project management in the planning phase. While in the design phase, there were many gaps among the analysis. In the implementation phase, researchers have focused on software development, and enterprise system significantly. In the testing phase, researchers clearly have focused on project management and there were a number of gaps. In the deployment phase, researchers have focused on project management, strategy, and methodology. Finally, in the maintenance phase, researchers have focused on Organization characteristics and Enterprise system selection process. 


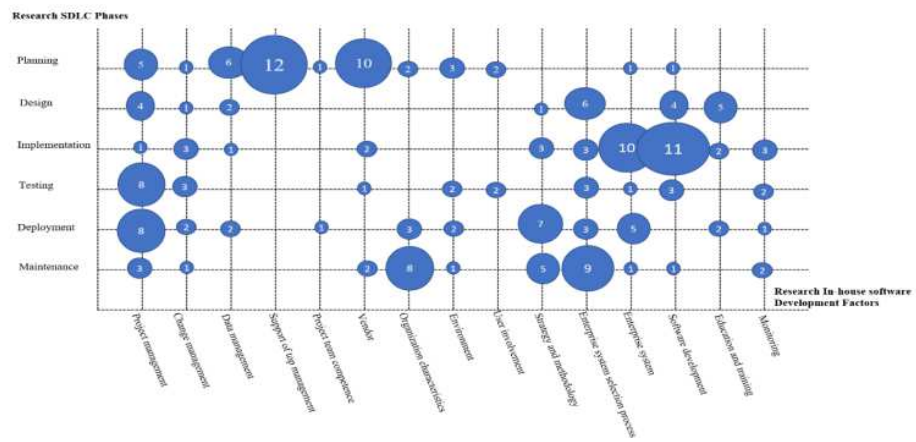

Figure 5: Bubble Systematic Snapshot Mapping Results

On the other hand, we categorized the gathered one hundred and two papers according to SDLC phases after conducting the bubble systematic snapshot mapping results. The results generated after removing the duplicates of the referenced factor many times in its sub-factors. Figure 6 shows that the highest number of research papers addressed the implementation phase (37 research papers). While the lowest number of research papers addressed the design phase (19 research papers). The requirements phase resulted in 34 research papers. The testing phase yielded 23 research papers. The deployment phase resulted in 32 research papers. Finally, the maintenance phase resulted in 23 research.

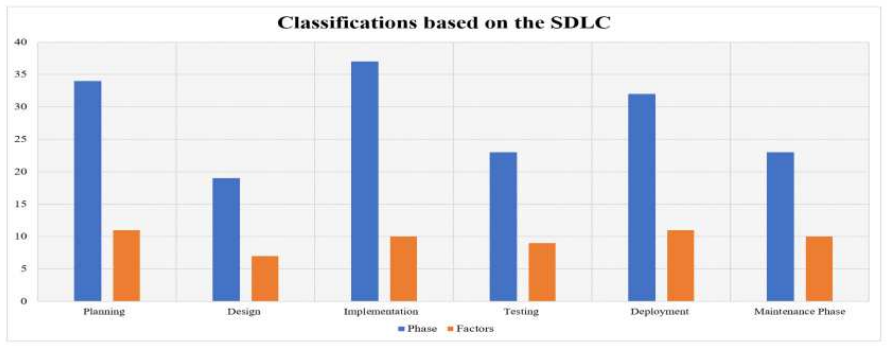

Figure 6: Classifications Based on the SDLC Findings

\subsection{Software Sized Classifications}

Recently, there has been a huge growth in the number of software enterprises, especially small and medium-sized enterprises (SME). This tendency compels the researchers to concentrate on studying representing models, frameworks, processes, tools, and techniques for SMEs projects from different perspectives. Based on the EU, a classification of software enterprises size is divided into two main types; large and SMEs. The large enterprises are classified according to the number of employees within the company which usually exceeds 250 employees. The SMEs are classified into three types (micro, small, small to medium); microenterprises usually employ less than 10 employees, small enterprises usually employ less than 50 employees. Finally, medium size enterprises usually employ employees more than 50 employees but less than 250 employees(Commission, 2003). In this part, we classified the one-hundred and two relevant papers based on the software enterprise size. Figure 7 shows that 50 articles carried their investigations in SMEs, 20 papers investigated the large systems, and the rest of the papers did not mention the size of software enterprise. 


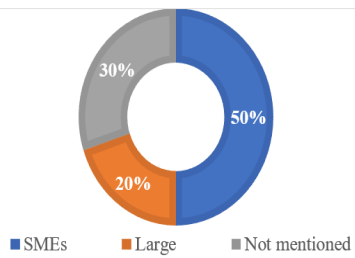

Figure 7: Classifications Based on Software Size

\subsection{Software Application List Findings}

This section shows a variety of application lists that can be used by sectors to name a few: information systems, business companies, universities, organizations, governments, healthcare, aircraft, and other sectors. These types meet a specific demand, and features that are specified by clients of custom-made software. For demonstrating the various usage of the custom-made software, we extracted the name of custom-made applications from the one hundred and two relevant papers. The findings show that 33 applications were investigated or researched between the periods from 2007 to 2018. This is shown in table 9 below.

Table 9: Classifications Based on Software Application Lists

\begin{tabular}{|c|c|c|c|}
\hline Application Name & Ref. & Application Name & Ref. \\
\hline $\begin{array}{l}\text { Custom E-Commerce } \\
\text { application }\end{array}$ & (Hrubeš; Schubert \& Merian-Str, 2015) & $\begin{array}{l}\text { Custom Sales Management } \\
\text { System }\end{array}$ & (Laar et al., 2014) \\
\hline $\begin{array}{l}\text { Custom Mobile } \\
\text { Application }\end{array}$ & (Fee, 2016) & $\begin{array}{l}\text { Higher Education Information } \\
\text { Systems }\end{array}$ & (Tomiwa, 2015) \\
\hline $\begin{array}{l}\text { Content Management } \\
\text { System }\end{array}$ & (Dick et al., 2011; Smith et al., 2009) & Unmanned Aircraft System & (Paulson et al., 2017) \\
\hline Custom ERP Projects & (Deep et al., 2008) & German Car Retailer & (Richert, 2017) \\
\hline Start-ups Project & (Bailetti, 2012; Johansson et al., 2016) & $\begin{array}{l}\text { Custom Computerized } \\
\text { AccountingSoftware }\end{array}$ & (Aduamoah, 2017) \\
\hline $\begin{array}{l}\text { E-Learning Bespoke } \\
\text { Solution }\end{array}$ & (Salem, 2012) & $\begin{array}{l}\text { Custom Laboratory Information } \\
\text { System }\end{array}$ & $\begin{array}{l}\text { (Sinard \& } \\
\text { Gershkovich, 2012) }\end{array}$ \\
\hline $\begin{array}{l}\text { E-portfolio of educational } \\
\text { system }\end{array}$ & (Matar, 2015) & $\begin{array}{l}\text { Custom Image Processing } \\
\text { Software }\end{array}$ & (Farooque et al., 2013) \\
\hline $\begin{array}{l}\text { Custom Product Life Cycle } \\
\text { Management System }\end{array}$ & (M. G. Wynn et al., 2008) & Bespoke Embedded System & (Perumal et al., 2013) \\
\hline $\begin{array}{l}\text { Custom Financial Services } \\
\text { Projects }\end{array}$ & (M. G. Wynn et al., 2008) & $\begin{array}{l}\text { Custom Smart Home } \\
\text { Management System }\end{array}$ & (Perumal et al., 2013) \\
\hline $\begin{array}{l}\text { Tailored Banks Enterprise } \\
\text { Applications }\end{array}$ & (Aranda et al., 2007) & $\begin{array}{l}\text { Home Routers Running Custom } \\
\text { Software }\end{array}$ & $\begin{array}{l}\text { (Sundaresan et al., } \\
\text { 2014) }\end{array}$ \\
\hline $\begin{array}{l}\text { Custom Management } \\
\text { Information Systems }\end{array}$ & (Duhan, 2007) & Custom Simulations & $\begin{array}{l}\text { (Papaloizou \& } \\
\text { Komodromos, 2009) }\end{array}$ \\
\hline Data Management Systems & (Forza \& Salvador, 2008) & $\begin{array}{l}\text { Specialist Autism Education } \\
\text { Projects }\end{array}$ & (Leach, 2010) \\
\hline Domestic Firm & (Kuivalainen et al., 2007) & Custom Defensive Solutions & (Pan \& Fung, 2011) \\
\hline Healthcare Specific System & (Ivarsson, 2018) & Night Vision System & $\begin{array}{l}\text { (Mateer \& Jones, } \\
\text { 2018) }\end{array}$ \\
\hline $\begin{array}{l}\text { Custom Customer } \\
\text { Relationship Management }\end{array}$ & $\begin{array}{l}\text { (Bass, 2016; Forza \& Salvador, 2008; Laakso, } \\
\text { 2017; M. Wynn et al., 2016) }\end{array}$ & $\begin{array}{l}\text { Custom Cloud-Based } \\
\text { Application }\end{array}$ & $\begin{array}{l}\text { (Rauschecker et al., } \\
\text { 2011; Saqib et al., } \\
\text { 2011) }\end{array}$ \\
\hline $\begin{array}{l}\text { Custom Web-Based } \\
\text { Systems }\end{array}$ & $\begin{array}{l}\text { (Abeywardena \& De Coster; Ciolkowski et al., } \\
\text { 2008; Lang \& Fitzgerald, 2007; Sulayman et al., } \\
\text { 2012; Waterman et al., 2013) }\end{array}$ & $\begin{array}{l}\text { Custom Service Management } \\
\text { Solution }\end{array}$ & (Ofoegbu et al., 2011) \\
\hline IoT Custom Solution & (Fafoutis et al., 2018) & & \\
\hline
\end{tabular}

\subsection{Years of Publish Findings}

This section shows the results according to the process of extractions on the year of publication of the one hundred papers between the years 2007 to 2018. The line graph in figure 8 shows the number of papers produced in the last twelve years. 


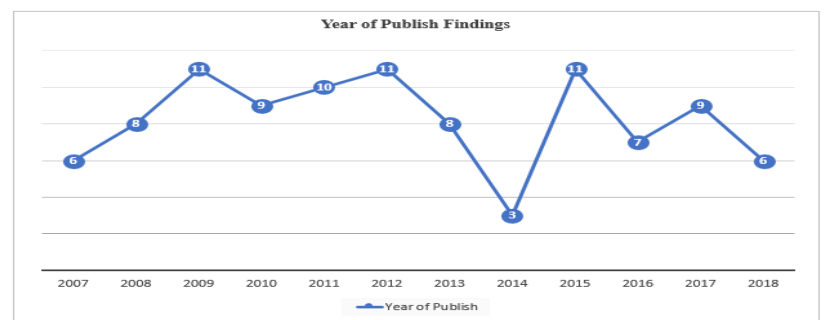

Figure 8: Classifications Based on Year of Publish

\subsection{Targeted Countries Findings}

In this section, we present the specific locations of the conducted 102 research studies. Table 10 shows the classification based on the target countries. 31 different target countries were the setting where the research was conducted. Interestingly the UK was highly represented in research for investigating different perspectives of custom-made software applications.

Table 10: Classifications Based on the Targeted Countries

\begin{tabular}{|c|c|c|c|}
\hline Country & $\#$ & Country & $\#$ \\
\hline UK & 14 & Switzerland & 2 \\
\hline Ireland & 6 & China & 2 \\
\hline India & 6 & Pakistan & 2 \\
\hline Sweden & 5 & Hong Kong & 2 \\
\hline Germany & 5 & Peru & 2 \\
\hline Canada & 4 & Romania & 2 \\
\hline Brazil & 3 & Luxembourg & 2 \\
\hline France & 3 & Thailand & 2 \\
\hline Austria & 3 & Georgia & 1 \\
\hline Malaysia & 3 & Tunisia & 1 \\
\hline Finland & 3 & Nigeria & 1 \\
\hline Cyprus & 3 & Israel & 1 \\
\hline Ghana & 3 & Czech & 1 \\
\hline USA & 3 & Denmark & 1 \\
\hline Colombia & 3 & Egypt & 1 \\
\hline Netherlands & 2 & & \\
\hline
\end{tabular}

In this part, we presented the extracted data in table 10 as a map graph which is shown in figure 9. The data revealed a dramatic change of colours on the map graph from dark green to light green. The dark green colour that represented the highest number of research papers investigating custom-made software. The light green colour represented the least number of research papers investigating custom-made software. UK fell on the dark green spectrum while Egypt fell on the light green colour spectrum.

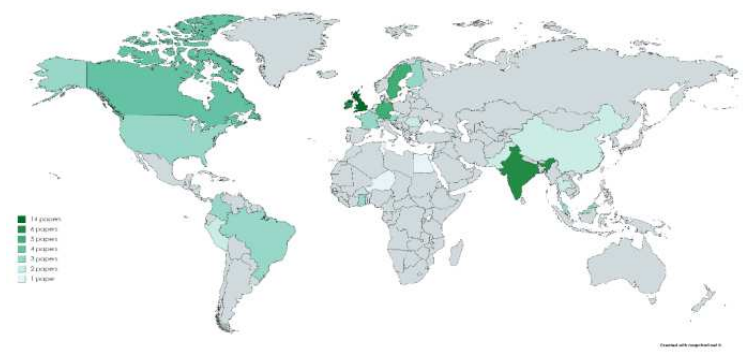

Figure 9: Map Graph for Targeted Countries 


\subsection{Top Ten Papers Citations Findings}

This classification shows the highest number papers cited by other researchers. The highest citation indicates a greater interest in the research as a research tool. It also indicates the tremendous quality level of the paper and findings. These increased citations pose as advantages that encourage other researchers to engage in an evidence-based research, to inform their own research process and to utilize the research findings in other fields of interest. The results for the top ten cited research papers are shown in table 11 below.

Table 11: Classifications Based on Top Ten Papers Citations

\begin{tabular}{|l|l|l|c|}
\hline \multicolumn{1}{|c|}{ Ref. } & Year & \multicolumn{1}{c|}{ Place of publish } & Citation No \\
\hline (Pino et al., 2008) & 2008 & Software Quality Journal & 288 \\
\hline (Petersen \& Wohlin, 2009) & 2009 & $\begin{array}{l}\text { International Symposium on Empirical Software Engineering and } \\
\text { Measurement }\end{array}$ & 197 \\
\hline (Laporte et al., 2008) & 2008 & European Conference on Software Process Improvement & 159 \\
\hline (Turner et al., 2010) & 2010 & International Journal of Project Management & 139 \\
\hline (Deep et al., 2008) & 2008 & Journals \& Books Journal of Manufacturing Technology Management & 136 \\
\hline (Svahnberg et al., 2010) & 2010 & Information and Software Technology & 136 \\
\hline (L. Xu \& Brinkkemper, 2007) & 2007 & European Journal of Information Systems & 134 \\
\hline (Savolainen et al., 2012) & 2012 & International Journal of Project Management & 133 \\
\hline (Kruchten, 2013) & 2013 & Journal of Software: Evolution and Process & 101 \\
\hline (Aranda et al., 2007) & 2007 & $\begin{array}{l}\text { 15th IEEE International Requirements Engineering Conference (RE } \\
\text { 2007) }\end{array}$ & 98 \\
\hline
\end{tabular}

\subsection{Research Methodology Findings}

In this part, our results indicate that a number of research papers used the conceptual method for the analysis of specific issues. Other research papers used the case study method either mainly to investigate a situation that has not been studied before or to describe the behaviour of practitioners during and after the implantation phase. A number of researchers used the qualitative approach to collect and analyse data about a particular phenomenon. Other researchers have used the quantitative method to collect and analyse data from a large group of people and to represent the results as statistics. Moreover, empirical studies used observation, experimentation, and other techniques to gather and analyse data. Additionally, a number of research papers used the literature review method in the process of gathering information. And, a number of research papers literally used the survey to gather information about a particular phenomenon. Figure 10 shows the statistical findings of the research methodologies used by the 102 relevant research papers.
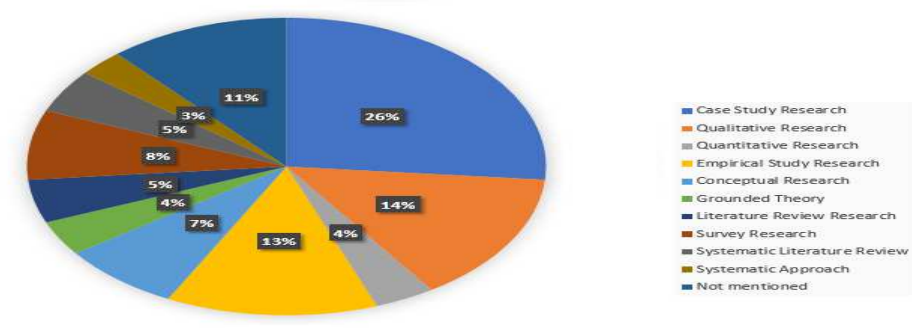

Figure 10: Classifications Based on Research Methodologies

\subsection{Research Publisher Findings}

This section contains the statistics of one hundred and two papers produced between the periods from 2007 to 2018 according to the name of the publishers. Table 12 presents the results of publishing classifications based on the publisher name. 24 publishers have published 55 journal papers and the highest number of papers were published by 
Elsevier, publisher of which were 16 journal papers.

Table 12: Classifications Based on Journals Publisher

\begin{tabular}{|l|c|l|c|}
\hline \multicolumn{2}{|c|}{ Publisher Name } & $\#$ & \multicolumn{2}{c|}{ Publisher Name } & $\#$ \\
\hline \multicolumn{3}{|c|}{ Journals } & 1 \\
\hline Elsevier & 16 & University of Limerick Institutional Repository & 1 \\
\hline Taylor \& Francis Online & 6 & ComSIS Consortium & 1 \\
\hline Emerald insight & 5 & Biodiversity Informatics & 1 \\
\hline Wiley online & 5 & IJCSIS & 1 \\
\hline Springer & 3 & TIM Review & 1 \\
\hline IGI Global & 2 & Inderscience & 1 \\
\hline IEEE & 2 & IJIRAE & 1 \\
\hline SERSC & 1 & IJETMAS & 1 \\
\hline NCBI & 1 & INFORMS & 1 \\
\hline WSCIT & 1 & Archives of Business Research & 1 \\
\hline Cambridge & 1 & RESAFE & \\
\hline Semantic scholar & 1 & & \\
\hline
\end{tabular}

As for the conference publishers, the number of relevant papers showed that 35 papers have been published in conferences. The highest number of papers, 13 papers in total, were published in the IEEE. The number of published papers slightly decreased to 8 papers in Springer, then it sharply fell down to 2 papers in the ACM and ESEM conferences and other publishers managed to include 1 paper only in conferences. Table 13 below shows the results for Classifications Based on Conferences Publishing.

Table 13: Classifications Based on Conferences Publisher

\begin{tabular}{|c|c|c|c|}
\hline Publisher Name & Ref & Publisher Name & Ref \\
\hline \multicolumn{4}{|c|}{ Conferences } \\
\hline IEEE & 13 & Environmental Informatics & 1 \\
\hline Springer & 8 & AISEL & 1 \\
\hline ESEM & 2 & OLKC & 1 \\
\hline $\mathrm{ACM}$ & 2 & eChallenges e-2011 & 1 \\
\hline University of Oxford & 1 & INASE & 1 \\
\hline e-Society & 1 & University of Gloucestershire & 1 \\
\hline iNCEB2010 & 1 & USENIX & 1 \\
\hline
\end{tabular}

As for analysis of report publishing, the relevant papers showed that 12 papers have been published by workshops or reports. These reports were presented as technical reports and others presented as theses in different universities. Results are shown in table 14.

Table 14: Classifications Based on Workshop, Reports Publisher

\begin{tabular}{|l|c|l|c|}
\hline \multicolumn{1}{|c|}{ Publisher Name } & $\#$ & \multicolumn{1}{c|}{ Publisher Name } & $\#$ \\
\hline \multicolumn{3}{|c|}{ Workshop, Reports } & 1 \\
\hline RESAFE & 1 & Utrecht University & 1 \\
\hline iNCEB2010 & 1 & Zarqa University & 1 \\
\hline Walden University & 1 & University of Ghana & 1 \\
\hline Aalto University & 1 & UWM: University of Wisconsin-Milwaukee & 1 \\
\hline Linköping University & 1 & NPS: Naval Postgraduate School & 1 \\
\hline ITGS & 1 & Masaryk University & \\
\hline
\end{tabular}

\subsection{Articles Indexed Findings}

In this section, figure 11 illustrates the statistical analysis of articles indexed to the journal paper ranking in terms of 39 Scopus ranked journals and other 18 journals. The number of identified 35 conference papers and the number of 12 reports. 


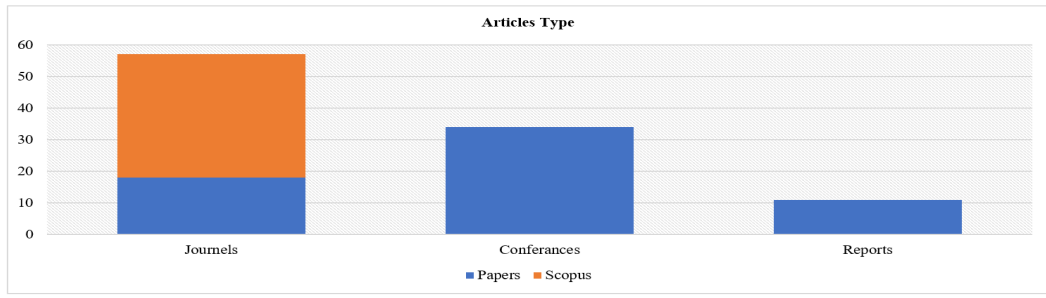

Figure 11: Classification Based on Article Types

\section{CONCLUSIONS}

We implemented a comprehensive systematic snapshot literature review on the custom-made software enterprises with an interest of development perspectives. A comprehensive research was used to find out the journal, conferences and reports, papers using the digital library search engines such as Elsevier, ACM, Springer Link, and IEEE Xplore in addition to the Google Scholar. We scanned a vast number of research papers using our inclusion and exclusion criteria which resulted in 102 papers relevant to the custom-made software. Following that, we classified these papers to the 9 identified associated classification schemas. This study resulted in that there was a high concentration of research interest in the implementation phase at the SDLC and fewer concentrations of research interest in the design and testing phases. The Bubble systematic snapshot mapping result revealed on the covered and uncovered contributions of 102 collected papers on the identified 15 factors. Some of these factors were covered by researchers, while other factors were not covered to be as a gap field and a research trend. Moreover, this study demonstrated that there was a higher research interest in the custom-made software at SMEs, a higher trend to use case study methodology, a high number of researches conducted in the UK and that the majority of articles were published in journals with indexed Scopus. Consequently, the significance part of this paper stems from the fact that it identified the common research trends to development perspectives in custom-made software enterprises based on the covered and uncovered systematic mapping contributions.

\section{REFERENCES}

1. Abeywardena, I. S., \& De Coster, R. Virtual Collaborative Framework for Managing Cross-cultural Software Projects.

2. Aduamoah, M. (2017). Riding the Waves of Technology: A Study into How SMEs Select Computerized Accounting Software (CAS) supplier in Developing Countries. Archives of Business Research, 5(10).

3. Akbar, R., Hassan, M. F., \& Abdullah, A. (2012). A framework of software process tailoring for small and medium size IT companies. Paper presented at the Computer \& Information Science (ICCIS), 2012 International Conference on.

4. Alamdy, S., \& Osman, R. (2017). Software Industry Practice in Africa: Case Study Sudan. Paper presented at the Computer Software and Applications Conference (COMPSAC), 2017 IEEE 41st Annual.

5. Allison, I. (2010). Organizational factors shaping software process improvement in small-medium sized software teams: A multi-case analysis. Paper presented at the Quality of Information and Communications Technology (QUATIC), 2010 Seventh International Conference on the.

6. Aranda, J., Easterbrook, S., \& Wilson, G. (2007). Requirements in the wild: How small companies do it. Paper presented at the Requirements Engineering Conference, 2007. RE'07. 15th IEEE International.

7. Arpaia, P., De Matteis, E., \& Inglese, V. (2015). Software for measurement automation: A review of the state of the art. Measurement, 66, 10-25. 


\section{Enterprises from the Development Perspectives}

8. Artz, P., Van De Weerd, I., \& Brinkkemper, S. (2010). Productization: The process of transforming from customer-specific software development to product software development. Department of Information and Computing Sciences Utrecht University, Utrecht, The Netherlands.

9. Artz, P., van de Weerd, I., Brinkkemper, S., \& Fieggen, J. (2010). Productization: transforming from developing customerspecific software to product software. Paper presented at the International Conference of Software Business.

10. Aslan, B., Stevenson, M., \& Hendry, L. C. (2015). The applicability and impact of Enterprise Resource Planning (ERP) systems: Results from a mixed method study on Make-To-Order (MTO) companies. Computers in Industry, 70, 127-143.

11. Bailetti, T. (2012). What technology startups must get right to globalize early and rapidly. Technology Innovation Management Review, 2(10).

12. Ballsun-Stanton, B., Ross, S. A., Sobotkova, A., \& Crook, P. (2018). FAIMS Mobile: Flexible, open-source software for field research. SoftwareX, 7, 47-52.

13. Barton, R., \& Thomas, A. (2009). Implementation of intelligent systems, enabling integration of SMEs to high-value supply chain networks. Engineering Applications of Artificial Intelligence, 22(6), 929-938.

14. Bass, J. M. (2016). Artefacts and agile method tailoring in large-scale offshore software development programmes. Information and Software Technology, 75, 1-16.

15. Bonomi Santos, J., \& Spring, M. (2013). New service development: managing the dynamic between services and operations resources. International Journal of Operations \& Production Management, 33(7), 800-827.

16. Ciolkowski, M., Heidrich, J., Simon, F., \& Radicke, M. (2008). Empirical results from using custom-made software project control centers in industrial environments. Paper presented at the Proceedings of the Second ACM-IEEE international symposium on Empirical software engineering and measurement.

17. Commission, E. U. (2003). Commission recommendation of 6 May 2003 concerning the definition of micro, small and medium-sized enterprises. Official Journal of the European Union, 46, 36-41.

18. Costache, D., Kalus, G., \& Kuhrmann, M. (2011). Design and validation of feature-based process model tailoring: a sample implementation of PDE. Paper presented at the Proceedings of the 19th ACM SIGSOFT symposium and the 13th European conference on Foundations of software engineering.

19. Deep, A., Guttridge, P., Dani, S., \& Burns, N. (2008). Investigating factors affecting ERP selection in made-to-order SME sector. Journal of Manufacturing Technology Management, 19(4), 430-446.

20. Deshpande, S., \& Richardson, I. (2009). Management at the outsourcing destination-global software development in India. Paper presented at the Global Software Engineering, 2009. ICGSE 2009. Fourth IEEE International Conference on.

21. Dick, M., Kern, E., Drangmeister, J., Naumann, S., \& Johann, T. (2011). Measurement and rating of software-induced energy consumption of desktop PCs and servers. Paper presented at the Innovations in sharing environmental observations and information. Proceedings of the 25th International Conference EnviroInfo October 5-7, 2011, Ispra, Italy.

22. Don, H., Hasselman, J., \& Wilbrink, A. (2011). Enterprise information systems as a service: re-engineering enterprise software as product-service system. Paper presented at the IFIP International Conference on Advances in Production Management Systems.

23. Duhan, S. (2007). A capabilities based toolkit for strategic information systems planning in SMEs. International Journal of Information Management, 27(5), 352-367. 
24. Dzamashvili Fogelström, N., Gorschek, T., Svahnberg, M., \& Olsson, P. (2010). The impact of agile principles on market $\square$ driven software product development. Journal of software maintenance and evolution: Research and practice, 22(1), 53-80.

25. Fabriek, M., van den Brand, M., Brinkkemper, S., Harmsen, F., \& Helms, R. (2008). Reasons for Success and Failure in Offshore Software Development Projects. Paper presented at the ECIS.

26. Fafoutis, X., Elsts, A., Piechocki, R., \& Craddock, I. (2018). Experiences and Lessons Learned from Making IoT Sensing Platforms for Large-Scale Deployments. IEEE Access, 6, 3140-3148.

27. Farooque, A. A., Chang, Y. K., Zaman, Q. U., Groulx, D., Schumann, A. W., \& Esau, T. J. (2013). Performance evaluation of multiple ground based sensors mounted on a commercial wild blueberry harvester to sense plant height, fruit yield and topographic features in real-time. Computers and electronics in agriculture, 91, 135-144.

28. Fee, S. B. (2016). 2.1. Reflections on Custom Mobile App Development for Archaeological Data Collection.

29. Fernández, D. M., Wagner, S., Lochmann, K., Baumann, A., \& de Carne, H. (2012). Field study on requirements engineering: Investigation of artefacts, project parameters, and execution strategies. Information and Software Technology, 54(2), 162-178.

30. Forza, C., \& Salvador, F. (2008). Application support to product variety management. International Journal of Production Research, 46(3), 817-836

31. Fricker, S. A. (2012). Software product management Software for People (pp. 53-81): Springer.

32. Gorschek, T., Gomes, A., Pettersson, A., \& Torkar, R. (2012). Introduction of a process maturity model for market $\square$ driven product management and requirements engineering. Journal of Software: Evolution and Process, 24(1), 83-113.

33. Gorschek, T., Tempero, E., \& Angelis, L. (2014). On the use of software design models in software development practice: An empirical investigation. Journal of Systems and Software, 95, 176-193.

34. Gray, S. (2011). Information Technology in a Global Society: Charleston.

35. Haug, A., Ladeby, K., \& Edwards, K. (2009). From engineer-to-order to mass customization. Management Research News, 32(7), 633-644.

36. Holland, C. P., Light, B., \& Gibson, N. (1999). A critical success factors model for enterprise resource planning implementation. Paper presented at the Proceedings of the 7th European conference on information systems.

37. Hrubeř, B. J. Comparison of development possibilities of information system: case study.

38. Ilyas, M., \& Khan, S. U. (2015). Software integration in global software development: Success factors for GSD vendors. Paper presented at the Software Engineering, Artificial Intelligence, Networking and Parallel/Distributed Computing (SNPD), 2015 16th IEEE/ACIS International Conference on.

39. Ilyas, M., \& Khan, S. U. (2017). Software integration in global software development: challenges for GSD vendors. Journal of Software: Evolution and Process, 29(8), e1875.

40. Iqbal, M. A., Zaidi, A. M., \& Murtaza, S. (2010). A new requirement prioritization model for market driven products using analytical hierarchical process. Paper presented at the Data Storage and Data Engineering (DSDE), 2010 International Conference on.

41. Ivarsson, A. (2018). Expediting Gathering and Labeling of Data from Zebrafish Models of Tumor Progression and Metastasis Using Bespoke Software. 


\section{Enterprises from the Development Perspectives}

42. Jebreen, I., Tamimi, M., Almajali, H., \& Janabi, F. (2018). Integration Testing in Small Packaged Software Vendors: A Systemic Snapshot. Paper presented at the Proceedings of the 2nd International Conference on E-Education, E-Business and E-Technology.

43. Jing, D., \& Yang, H. (2015). Creative computing for bespoke ideation. Paper presented at the Computer Software and Applications Conference (COMPSAC), 2015 IEEE 39th Annual.

44. Johansson, B., Deliallisi, B., \& Walraven, P. (2016). Exploring Choices of Software Sourcing Methods Among Start-Ups. Paper presented at the International Workshop on Global Sourcing of Information Technology and Business Processes.

45. Jones, R., \& Rowley, J. (2009). Presentation of a generic “EMICO” framework for research exploration of entrepreneurial marketing in SMEs. Journal of Research in Marketing and Entrepreneurship, 11(1), 5-21.

46. Kalus, G., \& Kuhrmann, M. (2013). Criteria for software process tailoring: a systematic review. Paper presented at the Proceedings of the 2013 International Conference on Software and System Process.

47. Kath, O., Schreiner, R., \& Favaro, J. (2009). Safety, security, and software reuse: a model-based approach. Paper presented at the Proceedings of the fourth international workshop in software reuse and safety.

48. Keil, M., \& Carmel, E. (1995). Customer-developer links in software development. Communications of the ACM, 38(5), 33-44.

49. Khurum, M., \& Gorschek, T. (2011). A method for alignment evaluation of product strategies among stakeholders (MASS) in software intensive product development. Journal of software maintenance and evolution: Research and practice, 23(7), 494516.

50. Kruchten, P. (2013). Contextualizing agile software development. Journal of Software: Evolution and Process, 25(4), 351-361.

51. Kuivalainen, O., Lindqvist, J., Saarenketo, S., \& Äijö, T. (2007). International growth of Finnish software firms: starting points, pathways and outcomes. Journal of Euromarketing, 16(1-2), 7-22.

52. Laakso, T. (2017). Customer Relationship Management Software Implementation in SMEs.

53. Laar, D., Konjaang, J., \& Tankia, B. (2014). Design and Development of a sales management system for SMEs in Northern Ghana. International Journal of Innovative Research in Advanced Engineering (IJIRAE), 10.

54. Lamersdorf, A., Munch, J., \& Rombach, D. (2009). A survey on the state of the practice in distributed software development: Criteria for task allocation. Paper presented at the Global Software Engineering, 2009. ICGSE 2009. Fourth IEEE International Conference on.

55. Danquah, EMELIA., \& Wireko, T. B. (2014). The impact of each element of emotional intelligence on customer service delivery: a customer satisfaction perspective. International Journal of Sales \& Marketing Management Research and Development, 4(2), 9-20.

56. Lang, M., \& Fitzgerald, B. (2007). Web-based systems design: a study of contemporary practices and an explanatory framework based on “method-in-action”. Requirements Engineering, 12(4), 203-220.

57. Laporte, C. Y., Alexandre, S., \& O'Connor, R. V. (2008). A software engineering lifecycle standard for very small enterprises. Paper presented at the European Conference on Software Process Improvement.

58. Leach, C. (2010). The use of Smartboards and bespoke software to develop and deliver an inclusive, individual and interactive learning curriculum for students with ASD. Journal of Assistive Technologies, 4(1), 54-57.

59. Lingannavar, R., \& Yammiyavar, P. G. A Frame Work for Innovation for the use by SMEs. 
60. Matar, N. (2015). Evaluating E-portfolio System Use within Educational Context. World of Computer Science \& Information Technology Journal, 5(3).

61. Mateer, J. E., \& Jones, R. W. (2018). Information Systems, Indirect Risks and Safety: An 8-Step Safety Management Process: IEEE ICIEA, Wuhan

62. Mijnhardt, F., Baars, T., \& Spruit, M. (2016). Organizational characteristics influencing SME information security maturity. Journal of Computer Information Systems, 56(2), 106-115.

63. Mishra, D., \& Mishra, A. (2009). Software process improvement in SMEs: A comparative view. Computer Science and Information Systems, 6(1), 111-140.

64. O'Connor, R. V., \& Coleman, G. (2009). Ignoring'Best Practice': why Irish software SMEs are rejecting CMMI and ISO 9000.

65. O'Connor, R. V., \& Laporte, C. Y. (2014). An innovative approach to the development of an international software process lifecycle standard for very small entities. International Journal of Information Technologies and Systems Approach (IJITSA), $7(1), 1-22$.

66. O'Connor, R. V., \& Laporte, C. Y. (2018). An innovative approach to the development of an international software process lifecycle standard for very small entities Computer Systems and Software Engineering: Concepts, Methodologies, Tools, and Applications (pp. 1300-1322): IGI Global.

67. O'Connor, R. V. (2014). Early stage adoption of ISO/IEC 29110 software project management practices: A case study. Paper presented at the International Conference on Software Process Improvement and Capability Determination.

68. Ofoegbu, A., Griffiths, M., \& Heinze, A. (2011). Themes and challenges for service management solutions in Small and Medium Enterprises (SMEs).

69. Pan, J. J. Y., \& Fung, C. C. (2011). Boutique Malware-Custom made attacks on e-business.

70. Papaloizou, L., \& Komodromos, P. (2009). Planar investigation of the seismic response of ancient columns and colonnades with epistyles using a custom-made software. Soil Dynamics and Earthquake Engineering, 29(11-12), 1437-1454.

71. Parry, S., Rowley, J., Jones, R., \& Kupiec-Teahan, B. (2012). Customer-perceived value in business-to-business relationships: A study of software customers. Journal of Marketing Management, 28(7-8), 887-911.

72. Paulson, C., Sobester, A., \& Scanlan, J. (2017). The rapid development of bespoke small unmanned aircraft: A Proposed Design Loop. The Aeronautical Journal, 121(1245), 1683-1710.

73. Perumal, T., Sulaiman, M. N., Sharif, K. Y., Ramli, A. R., \& Leong, C. Y. (2013). Development of an embedded smart home management scheme. International Journal of Smart Home, 7(2), 15-26.

74. Petersen, K., Feldt, R., Mujtaba, S., \& Mattsson, M. (2008). Systematic Mapping Studies in Software Engineering. Paper presented at the EASE.

75. Petersen, K., \& Wohlin, C. (2009). Context in industrial software engineering research. Paper presented at the Proceedings of the 2009 3rd International Symposium on Empirical Software Engineering and Measurement.

76. Pino, F. J., García, F., \& Piattini, M. (2008). Software process improvement in small and medium software enterprises: a systematic review. Software Quality Journal, 16(2), 237-261.

77. Ramasubbu, N., Cataldo, M., Balan, R. K., \& Herbsleb, J. D. (2011). Configuring global software teams: a multi-company analysis of project productivity, quality, and profits. Paper presented at the Software Engineering (ICSE), $201133 r d$ International Conference on. 


\section{Enterprises from the Development Perspectives}

78. Rauschecker, U., Meier, M., Muckenhirn, R., Yip, A. L. K., Jagadeesan, A. P., \& Corney, J. (2011). Cloud-based manufacturing-as-a-service environment for customized products.

79. Regnell, B., \& Brinkkemper, S. (2005). Market-driven requirements engineering for software products Engineering and managing software requirements (pp. 287-308): Springer.

80. Arora, Deepika., \& Saxena, A. (2013). Inter relationship of service quality aspects, customer satisfaction and customer loyalty in banking sector of india: a study of retail banking sector. International Journal of Research in Business Management, 1(4), $1-8$.

81. Richert, M. (2017). An energy management framework tailor-made for SMEs: Case study of a German car company. Journal of Cleaner Production, 164, 221-229.

82. Rodríguez-Dapena, P., \& Buitrago-Botero, M. F. (2015). How to certify the very small entity software processes using ISO/IEC 29110. Paper presented at the International Conference on Software Process Improvement and Capability Determination.

83. Rolandsson, B., Bergquist, M., \& Ljungberg, J. (2009). Open source programmer's strategies to cope with ideological tensions.

84. Rolandsson, B., Bergquist, M., \& Ljungberg, J. (2011). Open source in the firm: Opening up professional practices of software development. Research Policy, 40(4), 576-587.

85. Salem, A. (2012). Intelligent methodologies and technologies for e-Learning. Paper presented at the Emerging eLearning Technologies \& Applications (ICETA), 2012 IEEE 10th International Conference on.

86. Santos, G., Montoni, M., Vasconcellos, J., Figueiredo, S., Cabral, R., Cerdeiral, C., . . Rocha, A. R. (2007). Implementing software process improvement initiatives in small and medium-size enterprises in Brazil. Paper presented at the Quality of Information and Communications Technology, 2007. QUATIC 2007. 6th International Conference on the.

87. Saqib, S. M., Jan, M. A., Ahmad, B., Ahmad, S., \& Asghar, M. Z. (2011). Custom Software under the Shade of Cloud Computing. International Journal of Computer Science and Information Security, 9(5), 219.

88. Savolainen, P., Ahonen, J. J., \& Richardson, I. (2012). Software development project success and failure from the supplier's perspective: A systematic literature review. International journal of project management, 30(4), 458-469.

89. Sawyer, S. (2000). Packaged software: implications of the differences from custom approaches to software development. European journal of information systems, 9(1), 47-58.

90. Scacchi, W., \& Alspaugh, T. A. (2017). Achieving Better Buying Power for Mobile Open Architecture Software Systems Through Diverse Acquisition Scenarios.

91. Sceulovs, D., \& Gaile-Sarkane, E. The diversity of management theories for SME's development. Small, 10254(9732), 9970.

92. Schubert, P., \& Merian-Str, P. (2015). A Project Method for the Personalization of E-Commerce applications in SMEs.

93. Sinard, J. H., \& Gershkovich, P. (2012). Custom software development for use in a clinical laboratory. Journal of pathology informatics, 3 .

94. Smith, V. S., Rycroft, S. D., Harman, K. T., Scott, B., \& Roberts, D. (2009). Scratchpads: a data-publishing framework to build, share and manage information on the diversity of life. BMC bioinformatics, 10(14), S6.

95. Sulayman, M., Urquhart, C., Mendes, E., \& Seidel, S. (2012). Software process improvement success factors for small and medium Web companies: A qualitative study. Information and Software Technology, 54(5), 479-500. 
96. Shreedevi, d., \& Monisha, d. (2013). Web enabled customer care usability and monetary conversions.

97. Sundaresan, S., Burnett, S., Feamster, N., \& De Donato, W. (2014). BISmark: A Testbed for Deploying Measurements and Applications in Broadband Access Networks. Paper presented at the USENIX Annual Technical Conference.

98. Svahnberg, M., Gorschek, T., Feldt, R., Torkar, R., Saleem, S. B., \& Shafique, M. U. (2010). A systematic review on strategic release planning models. Information and Software Technology, 52(3), 237-248.

99. Tahir, T., Rasool, G., \& Noman, M. (2018). A Systematic Mapping Study on Software Measurement Programs in SMEs. EInformatica Software Engineering Journal, 12(1), 133-165.

100. Tamimi, M. (2018). AN EFFECTIVE RE-ENGINEERING MODEL FOR SMALL PACKAGED SOFTWARE. Zarqa University.

101. Tamimi, M., \& Jebreen, I. (2018). A Systematic Snapshot of Small Packaged Software Vendors' Enterprises. International Journal of Enterprise Information Systems (IJEIS), 14(2), 21-42.

102. Tauterat, T., Mautsch, L. O., \& Herzwurm, G. (2012). Strategic success factors in customization of business software. Paper presented at the International Conference of Software Business.

103. Tomiwa, B. D. (2015). Social Construction of Bespoke Software for Higher Education Management in a Sub-Saharan African Country. University of Ghana.

104. Turner, R., Ledwith, A., \& Kelly, J. (2010). Project management in small to medium-sized enterprises: Matching processes to the nature of the firm. International journal of project management, 28(8), 744-755.

105. Verma, A. (2010). Requirements analysis phase reengineering: Decision making \& requirements prioritisation among stakeholders for a new bespoke software project. Citeseer.

106. Wagner, D. S. (2017). Improving Bespoke Software Quality: Strategies for Application and Enterprise Architects.

107. Waterman, M., Noble, J., \& Allan, G. (2013). The effect of complexity and value on architecture planning in agile software development. Paper presented at the International Conference on Agile Software Development.

108. Wieczorkowski, J., Pawetoszek, I., \& Polak, P. (2015). Software standardization in the context of the innovativeness of enterprise operations. Paper presented at the P. Kommers, P. Isaias (red.), Proceedings of the International Conference eSociety.

109. Wynn, M., \& Turner, P. (2013). Effecting successful knowledge transfer: lessons from the UK Knowledge Transfer Partnership scheme. International Journal of Management in Education, 7(3), 293-312.

110. Wynn, M., Turner, P., Banik, A., \& Duckworth, G. (2016). The impact of customer relationship management systems in small business enterprises. Strategic Change, 25(6), 659-674.

111. Wynn, M. G., Shen, R., \& Brandao, R. (2008). Managing IS projects in SMEs-Tailoring the PRINCE2TM1 methodology.

112. Xu, L., \& Brinkkemper, S. (2007). Concepts of product software. European journal of information systems, 16(5), $531-541$.

113. Xu, P., \& Ramesh, B. (2008). Using process tailoring to manage software development challenges. IT Professional, 10(4). 


\section{AUTHOR'S DETAILS}

Moutasm Tamimi received his master's degree in software engineering at Zarqa University (ZU) with a cumulative average rating (excellent). With over seven years of industrial experience, he manages his own industry of packaged, custom software, and hybrid software development at (www.ITG7.com). In addition, he manages his own technical consultancy works at (www.IT-CRG.com). His research interests include software engineering, software maintenance, software process improvement in small companies, software quality, and research trends consultant. Contact him at tamimi@IT-CRG.com.

Fatimah Alghamdi is currently a master student of King Abdulaziz University, she received her bachelor's degree of the information system at King Khalid University (KKU) with a cumulative average rating (excellent). She works as a teacher assistant at Bisha University. She supervised on the women section of the e-learning unit at Bisha University and King Khalid University, Bisha Branch. Her research interests include secure software development life cycle, SMEs process improvements.

Ahid Yaseen is currently a master student of Zarqa University of software engineering; she received her bachelor's degree in software engineering at Zarqa University with first class honors. Her research interests are in Software Maintenance, Reverse Engineering, and component based Software Engineering quality. 
